\title{
Estudos de casos múltiplos: escolares com fissura labiopalatina no contexto da avaliação foniátrica
}

\author{
Multiple case studies: \\ schoolchildren with cleft lip and palate \\ in context of phoniatric evaluation
}

\section{Múltiplesestudios de caso: escolares conlábio leporino y paladar hendido en el contexto de la evaluación foniátrica}

\author{
Mônica Elisabeth Simons Guerra* (iD) \\ Vanessa Magosso Franchi* \\ Beatriz Cavalcanti de Albuquerque Caiuby Novaes*
}

\section{Resumo}

Introdução: A foniatria tem interesse em fissura labiopalatina (FLP) devido à complexidade em aspectos de fala, linguagem e audição. Objetivo: No contexto da avaliação foniátrica em crianças escolares com fissura labiopalatina, o objetivo do estudo de múltiplos casos foi descrever a interferência de fatores clínicos no desempenho em testes da função perceptiva auditiva de linguagem, assim como a associação entre eles. Método: Trinta crianças, entre 6 anos e 2 meses e 9 anos e 11 meses, foram avaliadas pelo médico foniatra, em serviço interdisciplinar de tratamento da FLP: 13 (43\%) fissura pósforame incisivo e 17 (57\%) fissura transforame incisivo. Foram descritos os fatores clínicos (incluindo tipo de fissura, idade na avaliação, sexo, condição socioeconômica, desempenho escolar, realização de fototerapia, exame audiológico e outros) e o desempenho em testes de discriminação auditiva, memória auditiva e consciência fonológica, incluindo a associação entre eles. Resultados: Não houve diferença significativa entre os grupos de fissura e médias de idade $(\mathrm{p}=0,618)$, sexo $(\mathrm{p}=0,431)$, nível socioeconômico $(\mathrm{p}=0,580)$ e desempenho escolar $(\mathrm{p}=0,785)$.A porcentagem de crianças que fez terapia é maior no grupo de fissura transforame $(94,1 \%)$. Alterações nos testes: 4 crianças $(13,3 \%)$ discriminação auditiva, 6 (20\%)

* Pontifícia Universidade de São Paulo - São Paulo, São Paulo, Brasil.

Contribuição dos autores:

MESG - concepção e delineamento do trabalho.

VMF - revisão crítica do conteúdo.

BCACN - aprovação da versão final e orientadora do mestrado.

E-mail para correspondência: Mônica Elisabeth Simons Guerra - simons.guerra@gmail.com Recebido: $13 / 03 / 2020$

Aprovado: $28 / 5 / 2020$ 
memória auditiva e 8 (26,7\%) consciência fonológica. Os casos com desempenho escolar insatisfatório, mais tempo de fonoterapia e piores médias do limiar de reconhecimento de fala tiveram desempenho pior em memória auditiva e consciência fonológica. Conclusão: A avaliação foniátrica possibilitou o apontamento de fatores clínicos da fissura, que parecem, de alguma forma, interferir no desenvolvimento da linguagem destas crianças.

Palavras chave: Fenda labial; Fissura palatina; Linguagem infantil.

\section{Abstract}

Introduction: Phoniatrics is interested in cleft lip and palate (CLP) due to the complexity in aspects of speech, language and hearing. Objective: In context of phoniatric evaluation in schoolchildren with CLP, the objective of multiple cases study was to describe the interference of clinical factors in the performance in tests of auditory perceptual function of language, as well as the association between them. Method: Thirty children, between 6 years and 2 months and 9 years and 11 months, were evaluated by Phoniatric doctor, in interdisciplinary treatment service for CLP: 13 (43\%) cleft palate and 17 (57\%) cleft lip and palate. Clinical factors (including type of cleft, age at evaluation, sex, socioeconomic status, school performance, speech therapy, audiological examination and others) and performance in tests of auditory discrimination, auditory memory and phonological awareness were described, including the association between them. Results: There was no significant difference between the cleft groups and the mean age $(p=0.618)$, sex $(p=0.431)$, socioeconomic level $(p=0.580)$ and school performance $(p$ $=0.785)$. The percentage of children who had attended therapy is higher in cleft lip and palate group (94.1\%). Changes in tests: $4(13.3 \%)$ auditory discrimination, $6(20 \%)$ auditory memory and $8(26.7 \%)$ phonological awareness. The cases with unsatisfactory school performance, more speech therapy time and worse speech recognition thresholds had a worse performance in auditory memory and phonological awareness. Conclusion: The phoniatric evaluation made it possible to identify clinical factors of the cleft, which seems, in some way, to interfere in the language development of these children.

Keywords: Cleft lip; Cleft palate; Child language.

\section{Resumen}

Introducción: Foniátrica está interesado en el labio leporino y paladar hendido debido a la complejidad en los aspectos del habla, el lenguaje y la audición. Objetivo: El contexto de la evaluación foniatría en escolares con labio leporino y paladar hendido, el objetivo del estudio de múltiples casos fue describir la interferencia de factores clínicos en el desempeño en las pruebas de la función auditiva perceptiva del lenguaje, así como la asociación entre ellos. Método: Treinta niños, entre 6 años y 2 meses y 9 años y 11 meses de edad, fueron evaluados por un médico foniátrico, en un centro de tratamiento interdisciplinario para labio leporino y paladar hendido: 13 (43\%) paladar hendido y 17 (57\%) labio leporino y paladar hendido. Se describieron y verificaron los factores clínicos (incluido el tipo de hendidura, la edad en la evaluación, el sexo, el estado socioeconómico, el rendimiento escolar, la fototerapia, el examen audiológico y otros) y el rendimiento en las pruebas de discriminación auditiva, memoria auditiva y conciencia fonológica, incluida la asociación entre ellos. Resultados: No hubo diferencias significativas entre los grupos de hendidura y la edad media $(\mathrm{p}=0.618)$, sexo $(\mathrm{p}=0.431)$, nivel socioeconómico $(\mathrm{p}=$ $0.580)$ y rendimiento escolar $(\mathrm{p}=0.785)$. El porcentaje de niños que se sometieron a terapia es mayor en el grupo de paladar hendido y labio leporino (94.1\%). Cambios en las pruebas: 4 niños $(13.3 \%)$ discriminación auditiva, $6(20 \%)$ memoria auditiva y 8 (26.7\%) conciencia fonológica. Los casos con un rendimiento escolar insatisfactorio, más tiempo de terapia del habla y peores umbrales de reconocimiento del habla tuvieron un peor rendimiento en la memoria auditiva y la conciencia fonológica. Conclusión: La evaluación foniátrica permitió identificar factores clínicos de la hendidura, que de alguna manera, parece interferir en el desarrollo del lenguaje de estos niños.

Palabras clave: Labio leporino; Fisura del paladar; Lenguaje infantil. 


\section{Introdução}

A foniatria estuda e cuida das crianças e adultos com distúrbios de linguagem e foi definida como área médica de atuação da otorrinolaringologia em 2006 pela Resolução do Conselho Federal de Medicina (CFM) ${ }^{1}$. Segundo Spinelli (1983), os distúrbios de linguagem se manifestam nas várias modalidades da emissão e da compreensão da linguagem, sendo que o grau de comprometimento é variável no que se refere a cada um dos aspectos e quanto à combinação entre eles ${ }^{2}$.

Os primeiros anos são fundamentais para o desenvolvimento da linguagem receptiva e expressiva, e depende de diversos fatores como genéticos, ambientais e emocionais, para estimular a neuroplasticidade e o aprendizado ${ }^{2,3}$. Não existe um ritmo de aquisição da linguagem único para todas as crianças, porém há alguns marcos da comunicação humana que devem ser seguidos ${ }^{2,3}$. Portanto, a Foniatria tem interesse nos pacientes com fissura labiopalatina devido à grande complexidade e variabilidade nos aspectos de produção de fala, desenvolvimento da linguagem e alterações na audição, desde os primeiros anos de vida, por falha na função dos músculos envolvidos no funcionamento velofaríngeo, e na aeração da tuba auditiva e alteração no crescimento crânio-facial ${ }^{4,5}$.

$\mathrm{O}$ fechamento velofaríngeo, que realiza a separação entre as partes oral e nasal da faringe, se faz por movimento craniodorsal do palato mole e por movimentos de medialização e anteriorização, respectivamente, das paredes laterais e posterior da faringe ${ }^{5}$. Nas crianças com fissura labiopalatina, antes da correção primária do palato, a disfunção velofaríngea está sempre presente por alterações displásicas durante o desenvolvimento embrionário dos músculos elevador do palato e tensor do véu palatino, e pode persistir após a cirurgia primária do palato. Essa disfunção é caracterizada por diversos sintomas com impacto no funcionamento adequado dos processos de fala, necessário para qualidade oral das vogais e das consoantes oclusivas e fricativas e sibilantes, como também no processo de deglutição e aeração do ouvido médio ${ }^{5,6}$.

Por consequência, primeiramente, a linguagem pode estar prejudicada por problemas de ordem anátomo-funcionais da fala, como distúrbios articulatórios e compensatórios, que envolvem emissão nasal e fraca pressão intraoral e se relacionam diretamente às alterações do palato e lábio, podendo persistir mesmo após cirurgias de correção do defeito facial ${ }^{4-6}$.

Por outro lado, privação de questões vivenciais como experiências sociais e afetivas também são importantes para construção da linguagem e são condições frequentes em famílias de crianças com problemas crônicos, tais como: internações hospitalares recorrentes e múltiplas cirurgias, dificuldades na sucção e deglutição, auto percepção do aspecto da face e da qualidade vocal e outros fatores comportamentais, e superproteção dos pais s, $^{4,7-12}$.

E, por fim, crianças com fissura labiopalatina apresentam alta frequência de alterações na função auditiva, por comprometimento da função do músculo tensor do véu palatino e elevador do palato que impede adequada abertura da tuba auditiva, gerando diminuição da aeração da orelha média, o que determina alta incidência de otites ${ }^{5,18-20}$. Kuo et al (2014) descrevem que antes de 1 ano, a otite média com efusão pode ocorrer pelo menos uma vez em $90 \%$ das crianças com fissura de palato ${ }^{18}$.

Alterações na audição podem impactar significativamente no desenvolvimento da função auditiva nas crianças com fissura palatina, sendo a otite média a causa mais comum de perda auditiva temporária nesta população ${ }^{13}$. Portanto, a otite média, no início da vida, pode levar a consequências em longo prazo em várias habilidades auditivas na idade escolar, mesmo após a recuperação clínica ${ }^{14}$. Sabemos, de acordo com a diretriz da clínica prática no manejo da otite média com efusão, que, esta pode ocorrer em qualquer criança de 2 meses de vida a 12 anos de idade, com ou sem deficiências ou outras condições que predisponham seu aparecimento e sua sequela. ${ }^{13}$ Portanto, a ligação entre audição e linguagem tem sido estudada frequentemente em crianças com perda auditiva flutuante leve a moderada decorrente de otite média, a qual pode comprometer as habilidades de linguagem, especialmente discriminação e consciência fonológica $^{13-17}$.

Nas crianças com fissura palatina, há consenso sobre a relação entre otite média, perda auditiva e desenvolvimento da linguagem, sendo que a perda auditiva condutiva transitória é uma das causas mais comum de alterações da linguagem nesta população ${ }^{18}$. Durante os primeiros anos de vida da criança, orienta-se que os médicos identifiquem, monitorem e gerenciem a otite média, desde a primeira efusão, possibilitando diagnóstico precoce. Os médicos devem, principalmente, informar aos 
pais sobre a história natural da doença e a importância do acompanhamento, além de determinar se a criança apresenta maior risco de problemas de fala, linguagem e/ou aprendizagem ${ }^{4,13}$.

Em muitos centros craniofaciais a recomendação para o tratamento da otite média relacionada à disfunção tubária na população com fissura labiopalatina é a cirurgia de timpanotomia para colocação do tubo de ventilação, uma vez que pode trazer benefício para a recuperação da audição evitando sequelas importantes em sua fala, linguagem ou aprendizagem $^{4,5,18,19}$. Outras condições da orelha média também podem ocorrer nesta população, com maior frequência nas crianças com fissura envolvendo o palato, como: otite aguda, otite crônica não supurativa, supurativa ou com colesteatoma e/ou sequelas cirúrgicas, como, por exemplo, perfuração da membrana timpânica. Em todos esses casos, é necessário tratamento específico para cada condição, podendo ocorrer, também, o efeito de perda auditiva permanente ${ }^{4,13}$.

$\mathrm{Na}$ criança com fissura labiopalatina, portanto, existem várias causas que podem afetar o desenvolvimento fonológico completo, afetando processamento de discriminação auditiva, consciência fonológica e desenvolvimento da leitura e escrita ${ }^{4,21}$. Nos últimos anos, estudos descrevem alterações nos aspectos que envolvem diversos níveis organizacionais do desenvolvimento de linguagem, tais como atraso de aquisição da fala nos primeiros anos de vida, formação deficitária de conteúdo lexical, falha na produção de sentenças complexas, desempenho insatisfatório de aprendizado de leitura e escrita, interferindo e dificultando a integração do indivíduo na sociedade ${ }^{4,7-11,21}$. E, também, identificaram variações significativas em funções corticais relacionadas com função linguística, como prejuízo em capacidades da memória, funções executivas e atencionais ${ }^{22,23}$.

No contexto da avaliação foniátrica em crianças escolares com fissura labiopalatina, o objetivo do presente estudo de múltiplos casos foi descrever a interferência de fatores clínicos no desempenho em testes da função perceptiva auditiva de linguagem, com enfoque em discriminação auditiva, memória auditiva e consciência fonológica, assim como a associação entre eles.

\section{Método}

Trata-se de um estudo de múltiplos casos, descritivo, derivado de dados parciais da dissertação de mestrado ${ }^{28} \mathrm{e}$ que foi aprovado pelo comitê de ética conforme o número de parecer 2403672.

O estudo foi realizado em serviço de saúde para diagnóstico e tratamento de pacientes com fissura labiopalatina, que atende o Sistema Único de Saúde (SUS), de natureza filantrópica. As 30 crianças selecionadas para o estudo são acompanhadas, desde o nascimento, por equipe interdisciplinar especializada e, no momento da coleta, foram atendidas pelo médico foniatra, em sala de consultório silenciosa, seguindo a avaliação foniátrica publicada no Tratado de Foniatria ${ }^{24}$.

Primeiramente, foram identificados os fatores clínicos de acordo com seguintes procedimentos: (1) Análise de prontuário para coleta das variáveis gênero, idade e escolaridade na avaliação, tipo de fissura, cirurgias primárias (queiloplastia e palatoplastia), cirurgia otológica para inserção do tubo de ventilação, e duração e aspectos de trabalho da fonoterapia. (2) Anamnese clínica com pais ou outro responsável para identificação de antecedentes da criança e antecedentes familiares em relação à linguagem e audição, relatos referentes ao desenvolvimento de fala e linguagem e perguntas referentes ao comportamento da criança, às queixa escolares e ao desempenho satisfatório da criança no ambiente escolar. (3) Análise sócio econômica, foi baseada na metodologia de classificação socioeconômica proposta pela autora Graciano (2013) ${ }^{25}$, utilizada neste serviço de saúde. (4) Avaliação audiológica para obter o limiar de reconhecimento de fala (LRF) em duas fases, no momento da coleta e por volta dos 3 anos de idade, por meio de dados do prontuário. (5) Exame otorrinolaringológico foi realizado no momento da consulta e foram descritos os aspectos da membrana timpânica (otoscopia).

A fim de manter o estudo o mais homogêneo possível, foram selecionadas apenas crianças com malformação do palato, por consequentemente disfunções dos músculos do fechamento velofaríngeo e da abertura da tuba auditiva, divididas em dois grupos; 17 (57\%) crianças com fissura transforame incisivo e 13 (43\%) crianças com fissura pós-forame. As crianças, juntamente com os responsáveis, foram esclarecidas quanto aos objetivos da pesquisa e de eventuais riscos, e consentiram a participação, e, por meio da análise de prontuário, foram excluí- 
das as crianças com perda auditiva sensorioneural, deficiência intelectual, deficiência visual moderada a grave e outras malformações ou síndromes associadas. Todos os indivíduos estavam matriculados em escola regular, do primeiro ao quarto ano do Ensino Fundamental I; a maioria pertencia à classe econômica no estrato baixo $(80 \%)$, com idade entre 6 anos e 2 meses e 9 anos e 11 meses (média de idade 8 anos e 2 meses) (desvio padrão de 2 anos e 2 meses), sendo que 14 (47\%) crianças eram do sexo masculino e 16 (53\%) crianças eram do sexo feminino.

Em seguida, as crianças foram submetidas, pelo médico foniatra, a 3 testes de função perceptiva da linguagem para avaliar a função perceptiva auditiva de linguagem: (1) discriminação auditiva padronizada para crianças de 5 a 9 anos e segundo sexo masculino e feminino, constituída de 30 pares de sílabas, sendo 10 pares iguais e 20 pares diferentes publicado por Rodrigues $(1981)^{24,26}$; (2) memória auditiva por sequência numérica segundo faixa etária ${ }^{24}$; (3) consciência fonológica por produção oral para crianças de 3 a 14 anos, classificadas nas categorias baixa, média e alta, e constituída pelas habilidades: síntese silábica, síntese fonêmica, rima, aliteração, segmentação silábica, segmentação fonêmica, manipulação silábica, manipulação fonêmica, transposição silábica e transposição fonêmica, publicado por Seabra e Capovilla (2012) ${ }^{27}$.

Por fim, análise estatística da amostra foi realizada em duas etapas: análise descritiva e associação estatística entre algumas variáveis.

A análise descritiva foi referente aos fatores clínicos (incluindo idade, sexo, nível socioeconômico, realização de terapia, cirurgias primárias, cirurgia de inserção de tubo de ventilação, alterações de comportamento, desempenho escolar, tipo de fissura, exame otoscópico e limiar de reconhecimento de fala aos 3 anos de idade e na coleta) e aos resultados do desempenho nos testes de percepção auditiva da linguagem (discriminação auditiva, memória auditiva e consciência fonológica). A associação estatística foi aplicada em tipo de fissura e resultados do desempenho dos testes de percepção auditiva da linguagem com algumas variáveis (fatores clínicos). As variáveis para as quais foi obtido um valor de $p$ menor que 0,200 nessa análise foram consideradas como variáveis explicativas no ajuste de um modelo de regressão logística multinominal (Hosmer e Lemeshow, 1989). No ajuste de um modelo de regressão logística binária no qual a memória auditiva é a variável resposta.

$\mathrm{O}$ tipo de fissura foi comparado por meio do teste t-Student com as médias da idade, aplicado o teste Quiquadrado para sexo, nível socioeconômico e desempenho escolar, e por meio do teste da razão de verossimilhanças com realização de terapia. $\mathrm{O}$ resultado do desempenho no teste de discriminação auditiva foi comparado por meio do teste t-Student com idade, e pelo teste de Mann-Whitney com LRF aos três anos e na coleta. O resultado do desempenho no teste de memoria auditiva foi comparado pelo teste t-Student com as médias da idade, pelo teste da razão de verossimilhanças com sexo, nível socioeconômico e desempenho escolar, e por meio do teste de Mann-Whitney com duração de fonoterapia e LRF aos três anos e na coleta. No ajuste de um modelo de regressão logística binária no qual a memória auditiva é a variável resposta.

Os dados da consciência fonológica foram publicados em Guerra e Novaes (2020 no prelo $)^{29}$.

\section{Resultados}

O estudo foi composto por 30 crianças, que durante avaliação médica foniátrica, mostraram ter boa atenção dialógica e boa interlocução.

Todas as crianças foram submetidas às cirurgias de correção palato e (ou) lábio primárias, sendo que a maioria das crianças $(90 \%)$ realizou estes procedimentos na idade adequada segundo protocolo do serviço de atendimento, a correção de lábio (queiloplastia) até 6 meses de idade e a correção do palato (palatoplastia) até os dois anos de idade.

Os dados descritivos obtidos na avaliação foniátrica estão representados nas Tabelas 1 e 2. De acordo com os dados do prontuário, todas as crianças do estudo foram avaliadas com profissional fonoaudiólogo ao longo do seu desenvolvimento, sendo que $25(83,3 \%)$ crianças foram submetidas à fonoterapia, em período que variou de 6 meses a 7 anos, por distúrbios articulatórios e compensatórios, por falha na pressão intraoral e, também, por omissões e (ou) substituições de fonemas (53,3\%) e fala ininteligível $(30 \%)$ de acordo com o relato dos pais e dados coletados no prontuário. 
Tabela 1. Resumo descritivo dos fatores clínicos observados na avaliação foniátrica em crianças com fissura labiopalatina: tipo de fissura, dados audiológicos e otológicos e resultados dos testes da função perceptiva auditiva de linguagem.

\begin{tabular}{|c|c|c|c|c|c|c|c|c|c|c|c|}
\hline criança & $\begin{array}{c}\text { tipo de } \\
\text { fissura } \\
*\end{array}$ & $\underset{* *}{\text { N. timp. }}$ & OD $* * *$ & OE $* * *$ & $\begin{array}{l}\text { LRF D } 3 \\
\text { anos }\end{array}$ & $\begin{array}{c}\text { LRF E } 3 \\
\text { anos }\end{array}$ & $\begin{array}{l}\text { LRF D } \\
\text { coleta }\end{array}$ & $\begin{array}{l}\text { LRF E } \\
\text { coleta }\end{array}$ & $\begin{array}{l}\text { Disc. } \\
\text { Aud. }\end{array}$ & $\begin{array}{l}\text { Mem. } \\
\text { Aud. }\end{array}$ & $\begin{array}{l}\text { Cons. } \\
\text { Fono. }\end{array}$ \\
\hline 1 & $\mathrm{~T}$ & 1 & $\mathrm{R}$ & $\mathrm{R}$ & 30 & 60 & 25 & 35 & $X$ & $x$ & B \\
\hline 2 & $P$ & 0 & $P$ & $\mathrm{P}$ & 45 & 35 & 50 & 40 & $x$ & $x$ & B \\
\hline 3 & $\mathrm{P}$ & 0 & $\mathrm{~N}$ & $\mathrm{R}$ & 20 & 55 & 10 & 40 & $x$ & $x$ & B \\
\hline 4 & $\mathrm{P}$ & 0 & $\mathrm{~N}$ & $\mathrm{~N}$ & 10 & 20 & 5 & 5 & $x$ & $x$ & B \\
\hline 5 & $\mathrm{~T}$ & 0 & $\mathrm{R}$ & $\mathrm{R}$ & 20 & 30 & 10 & 25 & & $x$ & B \\
\hline 6 & $\mathrm{~T}$ & 0 & $\mathrm{~N}$ & $\mathrm{~N}$ & 25 & 25 & 0 & 0 & & $x$ & B \\
\hline 7 & $\mathrm{~T}$ & 3 & $\mathrm{E}$ & $\mathrm{R}$ & 35 & 55 & 45 & 45 & & & B \\
\hline 8 & $\mathrm{~T}$ & 2 & TV & TV & 40 & 45 & 15 & 5 & & & B \\
\hline 9 & $\mathrm{~T}$ & 0 & $\mathrm{R}$ & $\mathrm{R}$ & 10 & 15 & 40 & 45 & & & $M$ \\
\hline 10 & $\mathrm{~T}$ & 1 & $\mathrm{~T}$ & $\mathrm{~T}$ & 15 & 30 & 10 & 20 & & & $M$ \\
\hline 11 & $\mathrm{~T}$ & 1 & $\mathrm{R}$ & $\mathrm{R}$ & 10 & 5 & 5 & 5 & & & $M$ \\
\hline 12 & $\mathrm{P}$ & 1 & $\mathrm{~T}$ & $\mathrm{~T}$ & 10 & 10 & 5 & 5 & & & $M$ \\
\hline 13 & $\mathrm{P}$ & 1 & $\mathrm{~N}$ & TV & 30 & 25 & 10 & 10 & & & $M$ \\
\hline 14 & $\mathrm{~T}$ & 1 & $\mathrm{~N}$ & $\mathrm{~N}$ & 20 & 5 & 10 & 10 & & & $M$ \\
\hline 15 & $\mathrm{P}$ & 0 & $\mathrm{~N}$ & $\mathrm{~N}$ & 5 & 15 & 5 & 5 & & & $M$ \\
\hline 16 & $\mathrm{P}$ & 0 & $\mathrm{~N}$ & $\mathrm{~N}$ & 30 & 55 & 5 & 5 & & & $M$ \\
\hline 17 & $\mathrm{~T}$ & 1 & $\mathrm{~T}$ & $\mathrm{~T}$ & 25 & 40 & 10 & 10 & & & $M$ \\
\hline 18 & $\mathrm{~T}$ & 0 & $\mathrm{~N}$ & $\mathrm{R}$ & 25 & 30 & 20 & 30 & & & $A$ \\
\hline 19 & $\mathrm{~T}$ & 2 & $\mathrm{P}$ & $P$ & 30 & 40 & 45 & 45 & & & $A$ \\
\hline 20 & $\mathrm{~T}$ & 3 & $P$ & $\mathrm{P}$ & 15 & 15 & 10 & 10 & & & A \\
\hline 21 & $P$ & 1 & $\mathrm{~T}$ & $\mathrm{~T}$ & 25 & 20 & 15 & 10 & & & A \\
\hline 22 & $\mathrm{P}$ & 2 & $\mathrm{R}$ & $\mathrm{R}$ & 30 & 30 & 10 & 10 & & & $A$ \\
\hline 23 & $\mathrm{P}$ & 2 & $\mathrm{R}$ & $\mathrm{R}$ & 15 & 15 & 15 & 5 & & & $A$ \\
\hline 24 & $\mathrm{~T}$ & 1 & $\mathrm{~N}$ & $\mathrm{~N}$ & 40 & 45 & 0 & 5 & & & A \\
\hline 25 & $\mathrm{P}$ & 0 & $\mathrm{~N}$ & $\mathrm{~N}$ & 10 & 10 & 10 & 10 & & & $A$ \\
\hline 26 & $P$ & 1 & $\mathrm{P}$ & $\mathrm{P}$ & 40 & 40 & 40 & 10 & & & $A$ \\
\hline 27 & $\mathrm{~T}$ & 2 & $\mathrm{~T}$ & $\mathrm{~T}$ & 40 & 40 & 10 & 10 & & & $A$ \\
\hline 28 & $\mathrm{P}$ & 2 & $\mathrm{~N}$ & $\mathrm{~N}$ & 20 & 5 & 10 & 5 & & & $A$ \\
\hline 29 & $\mathrm{~T}$ & 1 & $\mathrm{~N}$ & $\mathrm{~N}$ & 10 & 20 & 10 & 10 & & & $A$ \\
\hline 30 & $\mathrm{~T}$ & 0 & $\mathrm{R}$ & $\mathrm{R}$ & 5 & 5 & 25 & 25 & & & $A$ \\
\hline
\end{tabular}

Legenda: *Tipo de fissura labiopalatina: $\mathrm{T}$ - fissura transforame incisivo; $\mathrm{P}-$ fissura pos-forame incisivo. **Numero de timpanotomias para colocação de tubo de ventilação: 0 - não fez; 1 - 1 vez; 2 - 2 vezes; 3 - 3 vezes.***OD- otoscopia da orelha direita e OE -

otoscopia da orelha esquerda: N- normal; R - retração do tímpano; T- placa de timpanoesclerose; P - perfuração do tímpano; TV-

tubo de ventilação locado. LRF - limiar de reconhecimento de fala; D - direita; E - esquerda. Disc. Aud. - Discriminação auditiva: X

- alteração. Mem. Aud. - Memória Auditiva: X - alteração. Cons. Fono. - Consciência Fonológica: B - baixa (linhas coloridas em cinza claro=8), M - média (linhas coloridas em cinza médio=9); A - alta (linhas coloridas em cinza escuro=13). 
Tabela 2. Resumo descritivo dos dados da anamnese da avaliação foniátrica de crianças com fissura labiopalatina

\begin{tabular}{|c|c|c|c|c|c|c|c|c|c|}
\hline \multirow[b]{2}{*}{ Criança } & \multicolumn{3}{|c|}{ Antecedentes } & \multirow{2}{*}{ 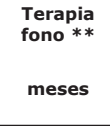 } & \multicolumn{2}{|c|}{$\begin{array}{c}\text { Atraso da fala } \\
\text { ( } 3 \text { anos de idade) }\end{array}$} & \multicolumn{3}{|c|}{ Relatos dos pais } \\
\hline & Perinatal & Cirúrgico & Familiar * & & $\begin{array}{c}\text { Omissões } \\
\text { substituições de } \\
\text { fonemas }\end{array}$ & $\begin{array}{c}\text { Fala } \\
\text { ininteligivel }\end{array}$ & $\begin{array}{c}\text { Desempenho } \\
\text { escolar } \\
\text { Insatisfatório }\end{array}$ & $\begin{array}{l}\text { Queixas } \\
\text { escolares }\end{array}$ & $\begin{array}{c}\text { Queixas do } \\
\text { comporta- } \\
\text { mento }\end{array}$ \\
\hline 1 & & & & & & & $x$ & $\mathrm{x}$ & $x$ \\
\hline 2 & $\mathrm{x}$ & & & 24 & $\mathrm{x}$ & $\mathrm{x}$ & $x$ & $\mathrm{x}$ & $x$ \\
\hline 3 & $x$ & & & & & & $x$ & $x$ & \\
\hline 4 & $x$ & $x$ & FIS & 48 & $x$ & $x$ & $x$ & $x$ & $x$ \\
\hline 5 & & & FIS DA & 50 & $x$ & $\mathrm{x}$ & & & \\
\hline 6 & & & & 84 & $x$ & $x$ & $\mathrm{x}$ & $\mathrm{x}$ & $\mathrm{x}$ \\
\hline 7 & $x$ & & DA LING & 48 & & & $\mathrm{x}$ & $\mathrm{x}$ & $\mathrm{x}$ \\
\hline 8 & $\mathrm{x}$ & & LING & 48 & $x$ & $x$ & $\mathrm{x}$ & $\mathrm{x}$ & $\mathrm{x}$ \\
\hline 9 & & $\mathrm{x}$ & FIS LING & 24 & $\mathrm{x}$ & $\mathrm{x}$ & $\mathrm{x}$ & $\mathrm{x}$ & $\mathrm{x}$ \\
\hline 10 & & & & 12 & & & & & \\
\hline 11 & & & & 60 & $\mathrm{x}$ & & $x$ & $\mathrm{x}$ & \\
\hline 12 & & & & 48 & $x$ & & $\mathrm{x}$ & $x$ & \\
\hline 13 & $x$ & $x$ & & 12 & & & & & $x$ \\
\hline 14 & & & & 72 & $x$ & $\mathrm{x}$ & & & \\
\hline 15 & $x$ & & & 6 & & & & $\mathrm{x}$ & \\
\hline 16 & & & & 6 & & & & & \\
\hline 17 & $\mathrm{x}$ & & & 12 & $\mathrm{x}$ & $\mathrm{x}$ & & & $\mathrm{x}$ \\
\hline 18 & & & & 18 & & & & & \\
\hline 19 & & & & 60 & & & & & \\
\hline 20 & & & LING & 36 & $x$ & & & & \\
\hline 21 & & & LING & & & & & & $x$ \\
\hline 22 & $x$ & & & 72 & $\mathrm{x}$ & $\mathrm{x}$ & $\mathrm{x}$ & $\mathrm{x}$ & \\
\hline 23 & & & & 60 & & & & & $\mathrm{x}$ \\
\hline 24 & & & & 24 & & & & & \\
\hline 25 & & & & 48 & $x$ & & & & \\
\hline 26 & & & FIS & & & & $\mathrm{x}$ & & $\mathrm{x}$ \\
\hline 27 & & & & 48 & $\mathrm{x}$ & & & & $\mathrm{x}$ \\
\hline \multicolumn{10}{|l|}{28} \\
\hline 29 & & $\mathrm{x}$ & & 6 & $\mathrm{x}$ & & & & \\
\hline 30 & & $\mathrm{x}$ & & 24 & $\mathrm{x}$ & & $\mathrm{x}$ & $\mathrm{x}$ & $\mathrm{x}$ \\
\hline$N(\%)$ & $\begin{array}{c}9 \\
(30 \%)\end{array}$ & $\begin{array}{c}3 \\
(10 \%)\end{array}$ & $8(26,7 \%)$ & $25(83,3 \%)$ & $16(53,3 \%)$ & $\begin{array}{c}9 \\
(30 \%)\end{array}$ & $\begin{array}{c}13 \\
(43,3 \%)\end{array}$ & $13(43,3 \%)$ & $\begin{array}{c}15 \\
(50 \%) \\
\end{array}$ \\
\hline
\end{tabular}

Legenda: * Antecedentes familiares: FIS - fissura labiopalatina, DA - deficiência auditiva e LING - linguagem. **fonoterapia. (Linhas coloridas são referentes ao resultado consciência fonológica: categoria baixa em cinza claro=8, categoria média em cinza médio $=9$ e categoria alta em cinza escuro=13).

Outros dados da avaliação foniátrica importantes para análise descritiva deste estudo foram: 9 $(30 \%)$ crianças apresentaram antecedentes perinatais, $5(16,7 \%)$ crianças foram submetidas a outros procedimentos cirúrgicos, excluindo queiloplastia e palatoplastia primárias, e $8(26,7 \%)$ crianças tiveram antecedente familiar para linguagem, deficiência auditiva e/ou fissura labiopalatina. De acordo com o relato dos pais, 13 (43,3\%) crianças tiveram desempenho escolar insatisfatório, 13 $(43,3 \%)$ tiveram queixa no ambiente escolar e 15 (50\%) tiveram queixa de alteração de comportamento. Em relação aos dados sobre a avaliação do exame físico otorrinolaringológico, todas as crianças $(100 \%)$ apresentavam palato corrigido pelo procedimento cirúrgico, $21(70 \%)$ crianças apresentaram alterações na otoscopia caracterizadas por membranas timpânicas com retração, perfuração, timpanoesclerose e inserção do tubo de ventilação. Verificou-se que $19(63,3 \%)$ crianças foram submetidas à timpanotomia para inserção de tubo de ventilação antes do terceiro ano de vida, sendo que destas, 6 crianças (20\%) foram submetidas a 2 procedimentos de colocação de tubo de ventilação na orelha média em tempos cirúrgicos diferentes, e 2 crianças $(6,7 \%)$ a 3 procedimentos em tempos cirúrgicos diferentes.

Aos 3 anos de idade, verificou-se que 17 $(56,7 \%)$ crianças apresentavam LRF alterado em ambas orelhas, $5(16,7 \%)$ crianças apresentavam LRF alterado em uma das orelhas e 7 (23,3\%) crianças não apresentavam alteração. E na cole- 
ta no momento da pesquisa, verificou-se que 7 $(23,3 \%)$ crianças apresentavam LRF alterado em ambas orelhas, $4(13,3 \%)$ crianças apresentavam LRF alterado em uma das orelhas. Portanto, o LRF estava alterado em $23(76,7 \%)$ crianças aos 3 anos de idade, e em 11 (36,7\%) crianças no momento da coleta.

A amostra de 30 crianças com fissura labiopalatina foi dividida em dois tipos de fissura com alteração no palato, sendo 13 (43\%) crianças com fissura pós-forame incisivo e 17 (57\%) crianças com fissura transforame incisivo.

Não houve diferença significativa quanto à distribuição das crianças entre os dois grupos de tipo de fissura e médias da idade $(\mathrm{p}=0,618)$, sexo ( $\mathrm{p}=0,431)$, nível socioeconômico $(\mathrm{p}=0,580)$, desempenho escolar $(\mathrm{p}=0,785)$ e do número de timpanotomias $(\mathrm{p}=0,379)$.

A maioria dos sujeitos fez terapia fonoaudiológica (Tabela 3), mas a porcentagem de indivíduos que fez terapia e que apresentam fissura transforame $(94,1 \%)$ é maior do que a porcentagem de indivíduos com fissura pós-forame $(69,2 \%)$. Não houve diferença significativa entre as porcentagens de indivíduos que fizeram terapia nos dois tipos de fissura, porém a diferença quase alcançou nível de significância $(p=0,066)$. O resumo descritivo do tempo de terapia dos 25 sujeitos que fizeram terapia é apresentado na Tabela 4.

Tabela 3. Distribuições de frequências e porcentagens da fonoterapia por tipo de fissura labiopalatina.

\begin{tabular}{ccccc}
\hline \multirow{2}{*}{ TIPO DE FISSURA } & \multicolumn{3}{c}{$\begin{array}{c}\text { Terapia } \\
\text { fonoaudiológica }\end{array}$} & Total \\
\cline { 2 - 4 } & Não & Sim & 13 \\
POS FORAME & 4 & $99,2 \%$ & $100 \%$ & 17 \\
TRANSFORAME & $30,8 \%$ & 16 & 100 & 30 \\
TOTAL & 1 & 94,1 & $100 \%$ \\
\hline
\end{tabular}

Não há diferença significativa entre as porcentagens de indivíduos que fizeram terapia nos dois tipos de fissura ( $p=0,066$ ).

Tabela 4. Resumo descritivo do tempo de fonoterapia (meses) por tipo de fissura lábio palatina e total.

\begin{tabular}{ccccccc}
\hline TIPO DE FISSURA & $\mathbf{N}$ & $\begin{array}{c}\text { Média } \\
\text { (meses) }\end{array}$ & Desvio padrão & $\begin{array}{c}\text { Mínimo } \\
\text { (meses) }\end{array}$ & $\begin{array}{c}\text { Mediana } \\
\text { (meses) }\end{array}$ & $\begin{array}{c}\text { Máximo } \\
\text { (meses) }\end{array}$ \\
\hline POS FORAME & 9 & 36,0 & 24,6 & 6 & 48 & 72 \\
TRANSFORAME & 16 & 39,1 & 23,1 & 6 & 42 & 84 \\
TOTAL & 25 & 38,0 & 23,2 & 6 & 48 & 84 \\
\hline
\end{tabular}

No estudo $25(83,3 \%)$ crianças que fizeram terapia, a o tempo de duração variou de 6 meses a 84 meses (7 anos).

Os resultados dos testes do desempenho da função perceptiva auditiva de linguagem estão representados na Tabela 1 . Nota-se que apenas $4(13,3 \%)$ crianças apresentaram desempenho abaixo do esperado para idade e sexo no teste de discriminação auditiva. No teste de memória auditiva sequencial por números, 6 crianças $(20 \%)$ apresentaram resultado abaixo do esperado para idade. E no teste de consciência fonológica, 8 $(26,7 \%)$ crianças apresentaram resultado categoria baixa, 9 crianças (30\%) apresentaram resultado ca- tegoria média e $13(43,3 \%)$ crianças apresentaram resultado categoria alta ${ }^{29}$.

A análise descritiva da discriminação auditiva, onde apenas 4 crianças $(13,3 \%)$ apresentaram alteração, não houve associação significativa entre discriminação auditiva com: idade $(p=0,483)$; LRF aos 3 anos de idade $(\mathrm{p}=0,142)$; LRF na coleta $(\mathrm{p}=0,180)$.

O desempenho no teste de memória auditiva foi associado com tipo de fissura, idade, sexo, nível socioeconômico, duração de fonoterapia, desempe- 
nho escolar e LRF aos três anos e na coleta (Tabela 5). As distribuições de porcentagens da memória auditiva nos dois tipos de fissura foram similares (Tabela 5), e não houve diferença significativa entre as distribuições de probabilidade nos dois tipos de fissura e memória auditiva $(\mathrm{p}=0,713)$ (teste da razão de verossimilhanças). Não houve diferença significativa entre as distribuições de probabilidade nos dois sexos $(\mathrm{p}=0,855)$ e nos níveis socioeconômicos $(\mathrm{p}=0,082)$. A média da idade observada no grupo com memória auditiva adequada foi maior que no grupo com memória não adequada (Tabela 6); no entanto, a diferença não foi significativa entre as médias da idade e categorias da memória auditiva $(\mathrm{p}=0,127)$ (teste $\mathrm{t}-\mathrm{Student})$. A maior média $(34,3$ meses) e a maior mediana (36 meses) de duração de fonoterapia foram observadas em crianças com desempenho inadequado no teste da memória auditiva, no entanto, não houve diferença significativa entre as distribuições de duração de fonoterapia entre as duas categorias de memória auditiva $(p=0,917)$ (teste de Mann-Whitney)(tabela 7). A porcentagem de crianças com memória auditiva adequada foi maior no grupo com desempenho escolar satisfatório $(94,1 \%)$, comparado com grupo com desempenho escolar insatisfatório. A diferença foi significativa $(p=0,024)$, ou seja, a probabilidade de memória auditiva adequada foi significativamente maior no grupo com desempenho escolar satisfatório (Tabela 8). As médias e medianas do LRF aos três anos de idade e na coleta foram piores nas crianças com memória auditiva não adequada do que nas crianças com memória auditiva adequada, no entanto, não houve diferenças significativas entre a memória auditiva e as distribuições do LRF aos 3 anos de idade $(p=0,212)$ e na coleta $(p=0,507)$ (teste de Mann-Whitney) (Tabela 9).

Tabela 5. Distribuições de frequências e porcentagens do desempenho na avaliação da memória auditiva por tipo de fissura

\begin{tabular}{ccc}
\hline \multirow{2}{*}{ TIPO DE FISSURA } & \multicolumn{2}{c}{ Memória auditiva adequada } \\
\cline { 2 - 3 } & NÃO & SIM \\
\hline \multirow{2}{*}{ PÓS-FORAME (N.13) } & 3 & 10 \\
TRANSFORAME (N.17) & $23,1 \%$ & $76,9 \%$ \\
TOTAL (N.30) & 3 & 14 \\
& $17,7 \%$ & $82,4 \%$ \\
& 6 & 24 \\
\hline
\end{tabular}

Não houve diferença significativa entre as distribuições de probabilidade nos dois tipos de fissura e memória auditiva ( $p=0,713$ ) (teste da razão de verossimilhanças).

Tabela 6. Distribuição das crianças com fissura labiopalatina no teste de memória auditiva de acordo com a idade na avaliação (meses)

\begin{tabular}{cccccccc}
\hline Testes & Categoria & N & $\begin{array}{c}\text { Idade Média } \\
\text { (meses) }\end{array}$ & $\begin{array}{c}\text { Desvio } \\
\text { padrão }\end{array}$ & $\begin{array}{c}\text { Mínimo } \\
\text { (meses) }\end{array}$ & $\begin{array}{c}\text { Mediana } \\
\text { (meses) }\end{array}$ & $\begin{array}{c}\text { Máximo } \\
\text { (meses) }\end{array}$ \\
\hline Memória & NÃO & 6 & 90,3 & 13,1 & 74 & 88 & 113 \\
auditiva & SIM & 24 & 100,4 & 11,6 & 73 & 101,5 & 119 \\
adequada & TOTAL & 30 & 98,4 & 12,4 & 73 & 101 & 119 \\
\hline
\end{tabular}

Não houve diferença significativa entre as médias da idade e categorias da memória auditiva $(p=0,127)($ teste t-Student). 
Tabela 7. Distribuição das crianças com fissura labiopalatina no teste de memória auditiva de acordo com a duração da fonoaterapia. (meses).

\begin{tabular}{cccccccc}
\hline $\begin{array}{c}\text { Teste / } \\
\text { Fonoterapia }\end{array}$ & Categoria & N & $\begin{array}{c}\text { Duração média } \\
\text { (meses) }\end{array}$ & $\begin{array}{c}\text { Desvio } \\
\text { padrão }\end{array}$ & $\begin{array}{c}\text { Mínimo } \\
\text { (meses) }\end{array}$ & $\begin{array}{c}\text { Mediana } \\
\text { (meses) }\end{array}$ & $\begin{array}{c}\text { Máximo } \\
\text { (meses) }\end{array}$ \\
\hline \multirow{2}{*}{$\begin{array}{c}\text { Memória auditiva } \\
\text { adequada }\end{array}$} & NÃO & 6 & 34,3 & 32,8 & 0 & 36 & 84 \\
& SIM & 24 & 31,0 & 24,2 & 0 & 24 & 72 \\
\hline
\end{tabular}

Não houve diferença entre as distribuições de duração de terapia nas duas categorias de memória auditiva ( $p=0,917)($ teste de MannWhitney).

Tabela 8. Distribuição das crianças com fissura labiopalatina no teste de memória auditiva de acordo com o desempenho escolar (satisfatório: sim ou não)

\begin{tabular}{cccc}
\hline $\begin{array}{c}\text { Desempenho escolar } \\
\text { satisfatório }\end{array}$ & \multicolumn{3}{c}{ Memória auditiva satisfatória } \\
\cline { 2 - 4 } NÃO & 5 & SIM & TOTAL \\
\hline \multirow{2}{*}{ SIM } & $38,5 \%$ & 8 & 13 \\
& 1 & $61,5 \%$ & 100 \\
& $5,9 \%$ & $94,1 \%$ & 17 \\
\hline
\end{tabular}

A porcentagem de crianças com memória auditiva adequada no grupo com desempenho escolar satisfatório ( $94,1 \%$ ) é maior que no grupo com desempenho insatisfatório. A diferença foi significativa $(p=0,024)$, ou seja, a probabilidade de memória auditiva adequada foi maior no grupo com desempenho escolar satisfatório (teste da razão de verossimilhanças).

Tabela 9. Distribuição das crianças com fissura labiopalatina no teste de memória auditiva de acordo com o limiar de reconhecimento de fala $(\mathrm{LRF}=\mathrm{dBNA})$ nas duas orelhas aos 3 anos de idade e na coleta.

\begin{tabular}{cccccccc}
\hline LRF & $\begin{array}{c}\text { Memória } \\
\text { auditiva } \\
\text { adequada }\end{array}$ & $\mathbf{N}$ & $\begin{array}{c}\text { Médio } \\
\text { dBNA }\end{array}$ & $\begin{array}{c}\text { Desvio } \\
\text { padrão } \\
\text { dBNA }\end{array}$ & $\begin{array}{c}\text { Mínimo } \\
\text { dBNA }\end{array}$ & $\begin{array}{c}\text { Mediana } \\
\text { dBNA }\end{array}$ & $\begin{array}{c}\text { Máximo } \\
\text { dBNA }\end{array}$ \\
\hline \multirow{2}{*}{$\begin{array}{c}\text { LRF 3 anos de } \\
\text { idade }\end{array}$} & NÃO & 6 & 31,3 & 11,4 & 15 & 31,25 & 45 \\
& SIM & 24 & 24,0 & 13,4 & 5 & 22,5 & 45 \\
\hline \multirow{3}{*}{ LRF na coleta } & Total & 30 & 25,4 & 13,1 & 5 & 25 & 45 \\
\hline & NÃO & 6 & 20,4 & 16,6 & 0 & 21,25 & 45 \\
& SIM & 24 & 15,2 & 12,7 & 2,5 & 10 & 45 \\
\hline
\end{tabular}

Não houve diferença entre a memória auditiva e as distribuições do LRF aos 3 anos de idade $(p=0,212)$ e na coleta $(p=0,507)($ teste de Mann-Whitney).

Os dados estáticos do desempenho nas habilidades de consciência fonológica foram descritos no artigo em análise para publicação de Guerra e Novaes $^{29}$. Não houve diferença significativa entre consciência fonológica e as distribuições de probabilidade em dois tipos de fissura, dois sexos e níveis socioeconômicos. A média maior da idade foi maior no grupo de categoria alta, e o desempenho escolar insatisfatório e o maior tempo de duração da fonoterapia foram mais frequentes no grupo de categoria baixa. E o resultado do LRF observado aos três anos de idade e na coleta foi pior para o grupo de crianças com resultados na categoria baixa e houve associação significativa apenas entre a consciência fonológica e o LRF aos 3 anos de idade (Guerra e Novaes, no prelo) ${ }^{29}$.

\section{Discussão}

O presente estudo avaliou crianças com fissura labiopalatina que, desde o nascimento, fizeram acompanhamento em um serviço de saúde de referência com equipe interdisciplinar especializada para tratamento das condições funcionais e anatômicas que a anomalia acarreta, e terapia direcionada a suas dificuldades de fala e linguagem.

Considerando-se a grande variabilidade das características clínicas apresentadas pelo grupo estudado e, portanto, a dificuldade para estabelecer-se um grupo controle, a discussão foi realizada referindo-se a dados da literatura.

A maioria das crianças (63\%) estudadas foi submetida à timpanotomia para inserção de tubo 
de ventilação antes dos 3 primeiros anos de vida, em conformidade com o descrito por Kuo e colaboradores (2014), que descreveram que a ocorrência de otite média com efusão é comum nos primeiros anos de vida de bebês com fissura que envolve o palato, podendo levar à perda auditiva condutiva $\mathrm{e}$, consequentemente, influenciar, negativamente, no desenvolvimento de linguagem ${ }^{18}$.

Briscoe, Bishop e Norbury (2001), reportaram que a função perceptiva auditiva é um processo complexo e alterações que afetam essa função podem levar a problemas na estrutura fonológica da linguagem, com consequentes efeitos adversos na aquisição do vocabulário e na alfabetização ${ }^{16}$. Os achados do presente estudo corroboram, de uma forma geral, as afirmações de Briscoe et al. (2001), particularmente no que se refere ao desempenho de $8(27 \%)$ crianças na consciência fonológica, $6(20 \%)$ crianças na memória auditiva, e $4(13 \%)$ crianças na discriminação auditiva. Observou-se, também, que houve associação direta entre o baixo desempenho nos testes de consciência fonológica e memória auditiva e os achados insatisfatórios quanto ao desempenho escolar insatisfatório, para $43,3 \%$ das crianças estudadas.

Muitos estudos demostraram prejuízo no aprendizado e baixo rendimento acadêmico na população com fissura labiopalatina associados a alterações em capacidades de funções linguísticas $7,9,10,11,22,23$. Do ponto de vista da Foniatria, observa-se que o desempenho escolar é reflexo de um efeito em cascata de várias situações e condições em que esta população está inserida e, que de alguma maneira, afeta a construção da linguagem ${ }^{4,5}$. Tabith Junior (2002) comenta que os fatores que interferem na função auditiva assim como os aspectos emocionais relacionados com estética (desfiguramento) facial, qualidade da fala e relações afetivas podem alterar o processo de construção de linguagem, sendo, portanto, essenciais para que a linguagem seja utilizada pela criança como um instrumento fundamental de relacionamento interpessoal, o que é importante para a adaptação escolar na infância, o ajustamento social na adolescência e o desempenho profissional e social na vida adulta ${ }^{4}$.

Observou-se, também, que as crianças na faixa de maior idade apresentaram resultado considerado alto no teste de consciência fonológica (acima de 8 anos e 11 meses) e memória auditiva adequada (acima de 8 anos e 4 meses). Esse desempenho sugere, primeiramente, que o fato destas crianças estarem em serviço de referência, onde se beneficiaram de fonoterapia de acordo com a demanda de cada indivíduo e com a finalidade de avaliar e orientar sobre os aspectos da fala e da linguagem, pode ter contribuído para o desempenho nas referidas faixas etárias. Outro fator importante a ser considerado como possível promotor deste melhor desempenho é o treinamento de consciência fonológica e memória auditiva (requisito, em muitas escolas, para o aprendizado da leitura e escrita), que possivelmente ocorreu durante a alfabetização ${ }^{27}$.

Destaca-se a criança 3 que, no momento da avaliação, tinha 9 anos e 5 meses e cursava o $4^{\circ}$ ano do ensino fundamental, e que apresentou alterações em todos testes aplicados. Essa criança, em particular, teve alterações perinatais, não fez fonoterapia e os pais relataram dificuldade escolar. Ela também apresentou retração de membrana timpânica à direita no exame otoscópico e resultado alterado do LRF, tanto aos 3 anos de idade quanto na coleta, e não foi submetida à timpanotomia para colocação de tubo de ventilação (apesar do histórico de alteração da audição). Aspectos importantes chamam atenção: os antecedentes pessoais podem ter influenciado no funcionamento neural de áreas cognitivas e nos resultados da função auditiva, interferindo, portanto, no processamento auditivo e, consequentemente, no amadurecimento biológico de complexas áreas da linguagem. Também, o fato desta criança não ter realizado a terapia fonoaudiológica preconizada, pode ter contribuído para seu pior desempenho nas habilidades de consciência fonológica já comprometida pelo histórico de alterações otológicas e audiológicas.

Neste estudo, a maioria das crianças $(83,3 \%)$ fez terapia e não houve diferença significativa entre os grupos de acordo com o tipo de fissura. A literatura ressalta que o tratamento cirúrgico do palato visa o fechamento da comunicação entre a cavidade oral e nasal e estabelece condições anatômicas para um funcionamento adequado do mecanismo velofaríngeo e da tuba auditiva, tendo como principal objetivo melhorar as condições de pressão intraoral e fluxo aéreo, porém, não implica necessariamente na melhora da produção da fala e da função da tuba auditiva; sendo necessário a terapia fonoaudiológica o mais precoce possível, que além da finalidade de prevenir ou corrigir alterações dos sons de fala, diminui também o impacto negativo do distúrbio da comunicação no desenvolvimento das habilidades linguísticas que podem refletir na 
alfabetização ${ }^{4,5,9}$. Portanto, observa-se nos resumos descritivos que crianças com pior desempenho nos testes tiveram tempo de duração da fonoterapia maior, o que sugere que crianças com maiores dificuldades exigiram terapias especializadas por várias demandas individuais.

No contexto da avaliação foniátrica, destaca-se a criança 6 (de 7 anos e 10 meses de idade), que foi o participante que fez fonoterapia por mais tempo, durante 7anos, e, mesmo assim, apresentou alterações nos testes de memória auditiva e de consciência fonológica. De acordo com o relato dos pais, a criança demonstrou atraso de linguagem (caracterizado por omissões e substituições de fonemas e fala ininteligível), teve dificuldade escolar e apresentou queixas comportamentais. No seu histórico clínico, aos 3 anos de idade, teve alteração do LRF em ambas as orelhas e, no momento da coleta, observou-se que o aspecto da membrana timpânica e o LRF encontravam-se dentro da normalidade, em ambas as orelhas. Sugere-se que a criança 6 apresentou questões comportamentais e cognitivas que influenciaram negativamente no desenvolvimento dos testes, visto que tanto a memória auditiva como a consciência fonológica são habilidades de funções corticais que dependem de fatores auditivos e cognitivos atencionais, e como também, de outros fatores externos emocionais.

$\mathrm{O}$ teste de discriminação auditiva foi realizado a partir da diferenciação de sons de fonemas em ambiente silencioso e teve melhor resultado comparado com os outros testes perceptivos auditivos. A discriminação auditiva é a capacidade de distinguir os sons da fala e é um processo complexo de diferenciação: ${ }^{1,24}$ neste sentido, era de se esperar piores resultados em uma população que apresenta histórico clínico de perda auditiva flutuante ou prolongada, como foi observado pela análise do LRF em dois momentos diferentes (aos 3 anos idade e na coleta). Uma das limitações no estudo, portanto, é que a análise da discriminação foi realizada apenas com sons de fonemas e não foram utilizados sons complexos ou palavras e, ainda, num ambiente silencioso com a proximidade do examinador (que seria fonte sonora) o que não necessariamente reflete a realidade de ambientes do cotidiano, na maioria ruidosa. Futuros estudos devem considerar os presentes achados e ampliar as situações de avaliação.

Das quatro crianças que apresentaram alteração em todos os testes, destaca-se a criança 4 , que tinha antecedente familiar de atraso de linguagem e caso de fissura labiopalatina, além de antecedente pessoal de afecções perinatais e atraso da fala. $\mathrm{O}$ LRF no momento da coleta e aos 3 anos de idade, bem como os aspectos da membrana timpânica estavam normais. Neste caso, a condição dos aspectos audiológicos e otológicos não foi relevante na determinação dos resultados baixos nas competências da percepção auditiva; porém outros elementos biológicos decorrentes de situações nos primeiros anos de vida, como os antecedentes pessoais, podem ter sido os agravantes na aquisição de linguagem e das funções cognitivas corticais.

De modo geral, a maioria das crianças do presente estudo, mesmo com alterações significativas em muitos critérios analisados, desempenhou resultados satisfatórios nos testes de função perceptiva auditiva. Estes resultados demonstraram a eficácia da intervenção precoce, do monitoramento constante do desenvolvimento da criança por profissionais especializados e também a eficácia das estratégias de tratamentos preconizadas no serviço de referência onde foi conduzido o estudo, sugerindo serem estes os aspectos protetores mais relevantes que forneceram a esta população mecanismos de adaptação no desenvolvimento das competências de linguagem. Portanto, o público alvo deste estudo representava crianças que tiveram o atendimento clínico e cirúrgico recomendados no tempo esperado, e nestas crianças, a consulta foniátrica também possibilitou a identificação de aspectos não diretamente relacionados à condição anátomo-funcional da fissura, mas que, de alguma forma, interferiram no desenvolvimento da linguagem destas crianças, sendo, portanto, uma avaliação clínica de grande relevância para essa população.

\section{Conclusão}

O presente estudo, no contexto da avaliação foniátrica, possibilitou a identificação de fatores clínicos da fissura, que podem interferir no desenvolvimento da linguagem destas crianças. A memória auditiva e a consciência fonológica foram os aspectos perceptivo-auditivos mais afetados, mas nem sempre explicou o desempenho escolar. Os antecedentes e (ou) alterações comportamentais de cada criança como indicadores de risco e ausência de terapia, em alguns casos, foram fatores explicativos no que se refere ao desempenho 
escolar, mesmo quando aspectos perceptivos não estavam alterados.

As características e a heterogeneidade da população estudada representam diferentes condições clínicas de crianças acompanhadas em um centro de referência especializado no tratamento de FLP. O bom desempenho da maioria dos sujeitos estudados sugere que a intervenção precoce, o monitoramento constante por profissionais especializados e as estratégias de tratamento preconizadas no serviço de referência são aspectos protetores em relação ao desenvolvimento das competências de linguagem.

\section{Referências bibliográficas}

1. Tabith Junior A, Favero ML, Pirana S. História da foniatria. In: Pirana S, Favero ML. Tratado de foniatria. 1 Ed. Rio de Janeiro: Thieme Revinter. 2020. p.1-4.

2. Spinelli M. Foniatria: Introdução aos distúrbios da comunicação. Audição / Linguagem. São Paulo. Editora Moraes. 1983.

3. Quirós JB e Gotter R. El lenguaje em el Niño. Buenos Aires. CEDIFA. 1964.

4. Tabith Junior A. Distúrbios da comunicação em portadores de fissuras labiopalatais: aspectos foniátricos. In: Mélega JM. Cirurgia plástica - Fundamentos e Arte. Rio de Janeiro: Editora Guanabara; 2002. p.25-8.

5. Tabith Junior A, Guerra MES. Fissuras labiopalatinas. In: Pirana S, Favero ML. Tratado de foniatria. Rio de Janeiro: Thieme Revinter; 2020. p.161-70.

6. Burg ML, Chai Y, Yao, CA, Magee W3rd, Figueiredo JC Epidemiology, Etiology, and Treatment of Isolated Cleft Palate. Front. Physiol. 2016; 7: 67.

7. Lamônica DAC, Silva-Mori MJF, Ribeiro CC, Maximino LP. Receptive and expressive language performance in children with and without cleft lip and palate. CoDAS. 2016; 28(4): 369-72.

8. Salas-Provance MB, Kuehn, DP, Marsh JL.Phonetic repertoire and syllable characteristics of 15 -month-old babies with cleft palate. J. Phonetics. 2003; 31(1): 23-38.

9. Chapman KL. The relationship between early reading skills and speech and language performance in young children with cleft lip and palate. The American Cleft Palate-Craniofacial Association. 2011; 48(3): 301-11.

10. Ha S. Effectiveness of a parent-implemented intervention program for young children with cleft palate. Int J Pediatr Otorhinolaryngol. 2015; 79(5): 707-15.

11. Priester GH, Goorhuis-Brouwer SM. Speech and language development in toddlers with and without cleft palate. Int J Pediatr Otorhinolaryngol. 2008; 72(6): 801-6

12. Fenha M, Santos EC, Figueira L. Avaliação das dimensões cognitivas e sócio-afectivas de crianças com fenda lábiopalatina. Psicologia: Saúde e Doenças. 2000; 1(1): 113-20.

13. Rosenfeld RM, Shin JJ, Schwartz SR, Coggins R, Gagnon L, Hackell JM et al. Clinical Practice guideline: otitis media with effusion (update). Otolaryngology - head and neck surgery. 2016; 154(IS) SI-S41.
14. Zumach A, Gerrits E, Chenault MN, Anteunis LJC. Otitis Media and Speech-in-Noise Recognition in School-Aged Children. Audiol Neurotol 2009; 14: 121-129.

15. Mody M, Schwartz RG, Gravel JS, Ruben RJ. Speech perception and verbal memory in children with and without histories of otitis media. J Speech Lang Hearing Res. 1999 Oct; 42: 1069-79.

16. Briscoe J, Bishop DVM, Norbury CF. Phonological processing, language and literacy: A comparison of children with mild-moderate sensorineural hearing loss and those with specific language impairment. J Child Psychol Psychiatry 2001; 42(3): 329-40.

17. Williams CJ, Jacobs AM. The impact of otitis media on cognitive and educational outcomes. Med J Aust. 2009; 191(9): 69-72.

18. Kuo CL, Tsao YH, Cheng HM, Lien CF, Hsu CH, Huang $\mathrm{CY}$, et al. Grommets for otitis media with effusion in children with clef palate: a systematic review. Pediatrics. 2014 Nov; 134(5): 983-94.

19. Tengroth B, Hederstierna C, Neovius E, Flynn T. Hearing thresholds and ventilation tube treatment in children with unilateral cleft lip and palate. Int J Pediatric Otorhinolaryngol. 2017; 97: 102-8.

20. Amaral MIR, Martins JE, Santos MFCS. Estudo da audição em crianças com fissura labiopalatina não-sindrômica. Braz $\mathbf{J}$ otorhinolaryngol. 2010; 76(2): 164-71.

21. Pamplona MDC, Ysunza PA. Total immersion speech camps for patients with cleft palate. Journal of Cleft lip palate and Craniofacial Anomalies. 2017 Nov; 4(3): 132-8.

22. Goldsberry G, O'leary D, Hichwa R, Nopoulos P. Functional abonormalities in the neural circuitry of Reading in men with nonsyndromic clefs of the li por palate. Clef palate craniofac J. 2006 Dec; 43(6): 683-90.

23. Lemos ICC, Feniman MR. Teste de Habilidade de Atenção Auditiva Sustentada (THAAS) em crianças de sete anos com fissura labiopalatina. Braz J Otorhinolaryngol. 2010 Mar-Apr; 76(2): 199-205.

24. Dualibi APF, Favero ML, Pirana S. A consulta Foniátrica. In: Pirana S, Favero ML. Tratado de foniatria. 1 Ed. Rio de Janeiro: Thieme Revinter. 2020. p.119-28.

25. Graciano MIG. Estudo Socioeconômico: Um instrumento técnico-operativo. São Paulo: Veras; 2013.

26. Rodrigues N. Neurolinguística dos distúrbios da fala. São Paulo: Editora Cortez; 1992.

27. Seabra AG, Capovilla FC. Teste de consciência fonológica por produção oral. In: Seabra AG, Dias NM. Avaliação neuropsicológica cognitiva: linguagem oral. Vol 2. São Paulo: Memnon; 2012. p.117-22.

28. Guerra MES. Crianças com fissura labiopalatina: relação entre percepção auditiva e características audiológicas e otológicas no contexto da avaliação foniátrica (dissertação). São Paulo: Pontifícia Universidade de São Paulo; 2019.

29. Guerra MES, Novaes BCAC. Clef lip and Palate: relationship between phonological awareness and audiological characteristics in children. Cleft Palate Craniofac J. no prelo 2020. 